\title{
Assembly and variation of root-associated microbiota of rice during their vegetative growth phase with and without lindane pollutant
}

\author{
Jiayin Feng ${ }^{1,2}$, Ashley E. Franks ${ }^{3,4}$, Zhijiang $\mathrm{Lu}^{1,2}$, Jianming $\mathrm{Xu}^{1,2}$, Yan $\mathrm{He}^{1,2, *}$ \\ 1 Institute of Soil and Water Resources and Environmental Science, College of Environmental and Resource Sciences, Zhejiang University, \\ Hangzhou 310058, China \\ 2 Zhejiang Provincial Key Laboratory of Agricultural Resources and Environment, Hangzhou 310058, China \\ 3 Department of Physiology, Anatomy and Microbiology, La Trobe University, Bundoora, Victoria, 3086, Australia \\ 4 Centre for Future Landscapes, La Trobe University, Bundoora, Victoria, 3086, Australia
}

H I G H L I G H T S

- Rice microbiota responded to lindane pollutant was studied spatiotemporally.

- Growth time, soil types and rhizo-compartments had significant influence.

- Lindane stimulated the endosphere microbiota of rice which was highly dynamic.

- Root-soil-microbe interactions induced an inhibited redox-coupled lindane removal.

- This work was beneficial to better regulation of plant growth against adversity.

\section{ARTICLE INFO}

Article history:

Received May 29, 2020

Revised August 24, 2020

Accepted September 9, 2020

Keywords:

Lindane pollutant

Rice (Oryza sativa L.)

Root-associated microbiota

Root-microbe-soil interaction

Vegetative growth phase

Metagenome functions
GRAPHICAL ABSTRACT

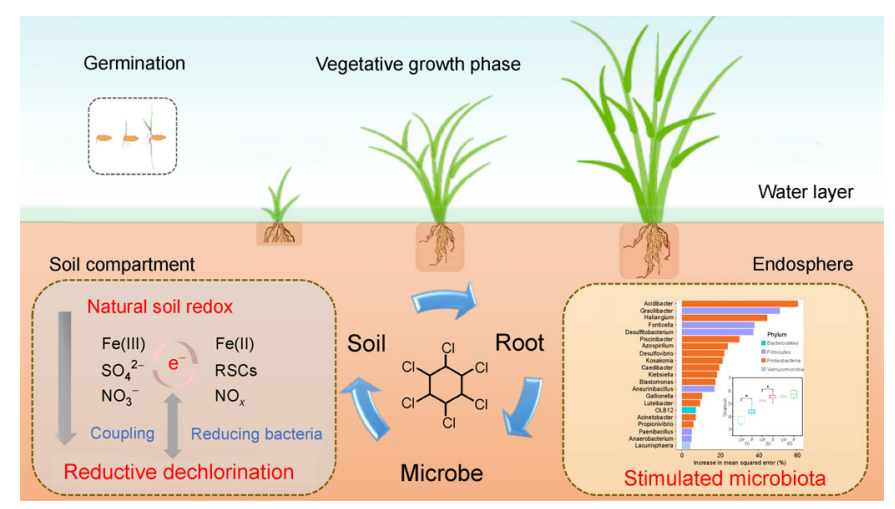

A B S TR A C T

Soil-derived microbiota associated with plant roots are conducive to plant growth and stress resistance. However, the spatio-temporal dynamics of microbiota in response to organochlorine pollution during the unstable vegetative growth phase of rice is not well understood. In this study, we focused on the rice (Oryza sativa L.) microbiota across the bulk soil, rhizosphere and endosphere compartments during the vegetative growth phase in two different soils with and without lindane pollutant. The results showed that the factors of growth time, soil types and rhizo-compartments had significant influence on the microbial communities of rice, while lindane mostly stimulated the construction of endosphere microbiota at the vegetative phase. Active rice root-soil-microbe interactions induced an inhibition effect on lindane removal at the later vegetative growth phase in rice-growth-dependent anaerobic condition, likely due to the root oxygen loss and microbial mediated co-occurring competitive electron-consuming redox processes in soils. Each rhizocompartment owned distinct microbial communities, and therefore, presented specific ecologically functional categories, while the moderate functional differences were also affected by plants species and residual pollution stress. This work revealed the underground micro-ecological process of microbiota and especially their potential linkage to the natural attenuation of residual organochlorine such as lindane.

(c) Higher Education Press 2020

* Corresponding author
E-mail address: yhe2006@zju.edu.cn (Y. He) 


\section{Introduction}

Plants assemble microbial populations in their rhizosphere from the surrounding soil though the production of root exudates and modification of soil chemistry. Rhizosphere associated microbes can be helpful for plant growth, nutrient uptake, and resistance to environmental stresses (Castrillo et al., 2017; Luo et al., 2017; Zhang et al., 2019; Xu et al., 2020a). Biotic and abiotic factors significantly influence the structure and function of root-associated microbial community, which can impact the benefit to the plant (Bulgarelli et al., 2012; Santos-Medellín et al., 2017; Dai et al., 2018). Advances in sequencing technologies are allowing in depth examination of the confounding factors that drive assembly of the rhizosphere associated community.

In the rhizosphere of plants, soil type, physiochemical characteristics and plant genomics all impact the structure of the final community. Soil characteristics have the greater impact on the structure of the rhizosphere associated community than host genotypes (Edwards et al., 2015). Drought stress makes for a reconstruction of root microbiota of rice which can contribute to plant survival under extreme environmental conditions (Santos-Medellín et al., 2017). The rhizosphere associated community is also crucial for plant development with root growth, and the microbial communities shift temporally over time and provide protection against external stresses (Edwards et al., 2018; Zhang et al., 2019). Host genetics still have important impacts with hybrid rice containing a more stable rhizosphere microbiota, and having more resilience, than conventional cultivars when under organochlorine pollution (Feng et al., 2019). Understanding changes to the plant-microbiota spatio-temporal interactions in response to environmental stresses during the vegetative growth phase is essential for resilience in situations of environmental stress. However, this kind knowledge is far less reached with respect to environmental pollution stress, especially organic pollution stress, as compared with those well-down regarding nutrient and water stress (SantosMedellín et al., 2017; Zhang et al., 2019).

Before being banned 30 years ago, hexaclorocyclohexanes $(\mathrm{HCHs})$ were used worldwide in protecting crops as a typical organochlorine pesticide (OCP) (Niu et al., 2013; Xu et al., 2020b). Given the stable aromatic ring structure, low water solubility and high toxicity, their residues continue to present a persistent and broad risk to environment (Xu et al., 2015; Salam et al., 2017). China was ever the major producer of $\mathrm{HCHs}$, and contributed over 4 tons to the whole world (Hua and Shan, 1996). Residual concentrations of $\mathrm{HCH}$ ranging from 0.146 to $23.9 \mathrm{\mu g} \mathrm{kg}^{-1}$ have been reported in Chinese agricultural soils (Niu et al., 2013); some higher residual contents were also verified afterwards, e.g., a content of 42.3 $\mu \mathrm{g} \mathrm{kg}^{-1}$ had been reported by Chen et al. (2015) in vegetable soils of Guangzhou, China. Other countries reported the high residue of $\mathrm{HCH}$ even up to over $20 \mathrm{mg} \mathrm{kg}^{-1}$ in soils, such as India and Thailand (Poolpak et al., 2008; Chouychai et al., 2015). As an important $\mathrm{HCH}$ isomer, lindane $(\gamma-\mathrm{HCH})$ shows the exclusive insecticidal activity, and is still modestly used in some specific fields and districts (Tang et al., 2016). It is one of the most representative organochlorine pollutants for investigation of the below-ground micro-ecological impact of residual OCPs on crop growth, and thereby on agricultural production.

Rice is an important crop around the world (Zhang et al., 2019). Soils for rice cultivation are largely anaerobic during the growing season due to continuous flooding (KögelKnabner et al., 2010). Under waterlogged anaerobic soil environments, the dissipation of OCP is mostly through the reductive dechlorination pathway. Reduction of OCP is coupled to natural soil redox reactions due to competition for electron donors and acceptors (Xue et al., 2017; Zhu et al., 2019). Radial $\mathrm{O}_{2}$ loss (ROL) by rice roots forms a narrow oxygenated zone in the rhizosphere that affects the soil biogeochemistry and microbial community function (Wu et al., 2012). Our previous research has suggested that rice root growth suppressed the dechlorinated dissipation of OCPs through the ROL and disruption of the anaerobic environment required for dichlorination (Feng et al., 2019, 2020a); however, this finding still needs to be further verified as it is based on a static observation of a single time point. The rhizosphere associated microbial community dynamically changes during root growth and responds to OCPs stress, both of which have potential impacts to OCP degradation.

In this work, we report on the impact of lindane on the bulk soils, rhizosphere and endosphere associated microbial communities due to rice (Oryza sativa L.) cultivation in two different soils. We evaluate the effects of pollution, soil type, growth time and rhizo-compartment, separately or interactively, in regulating the assembly and variation of rice rootassociated microbiota during rice vegetative growth phase. This evaluation provides insight into microbial processes linkage to the natural attenuation of residual lindane as a OCP. We hypothesized that: 1) there would be a significant interaction effect of soil types, rice growth time, rhizocompartments, and lindane pollution on the assembly of rice root-associated microbiota; 2) OCP pollution would impact on the root microbiota, with the effect dynamically dependent on the resistance of rice root during growth; 3) the reductive dechlorination of OCP in rhizosphere soils would be interrupted by rice growth, with the scale of impact dependent on growth time due to the $\mathrm{O}_{2}$ dynamic secretion properties of the root.

\section{Material and methods}

\subsection{Soils}

Surface soils (top $20 \mathrm{~cm}$ ) were sampled from two different agricultural paddocks in Jiaxing, Zhejiang Province $\left(30^{\circ} 50^{\prime}\right.$ 8.74" N, 12043'3.68" E) (soil 1, S1) and Yingtan, Jiangxi Province of China $\left(28^{\circ} 13^{\prime} 35.12^{\prime \prime} \mathrm{N}, 116^{\circ} 53^{\prime} 54.25^{\prime \prime} \mathrm{E}\right)$ (soil 2, S2). The lands are representative for the rice production in Yangtze River delta of China. Soil samples were collected by 
"S" sampling strategy in a $20 \mathrm{~m} \times 20 \mathrm{~m}$ field, transported back to the greenhouse, air-dried and passed through a 2-mm sieve for batch experiments. Both soil 1 and soil 2 were detected without lindane. Other basic soil properties, such as organic matter, phosphorus, $\mathrm{Fe}(\mathrm{II})$ and $\mathrm{pH}$ are listed in Table 1. To clearly investigate changes in assembly of root-associated microbiota in response to pollution stress of soil residual organochlorine such as lindane, we also set a little bit higher spiked concentration as compared to usually reported in the previous literatures, to expand pollution effect at the most while still allow the rice to grow (Rani et al., 2018; Feng et al., 2020b). Samples were divided in half. One half was spiked with lindane (around $50 \mathrm{mg} \mathrm{kg}^{-1}$ ) dissolved in $20 \mathrm{~mL}$ acetone, mixed thoroughly, evaporated for $24 \mathrm{~h}$ in a fume hood; and the other half was treated as the same procedure, only with the exception of lindane addition. After that, both spiked and nonspiked soils were separately maintained at a flooded condition through addition of deionized water to $2 \mathrm{~cm}$ above the soil for 7 days at room temperature. Lindane was then measured as outline below and found to have initial concentrations of 33.6 $\mathrm{mg} \mathrm{kg}^{-1}$ and $34.0 \mathrm{mg} \mathrm{kg}^{-1}$ in soil 1 and 2 respectively.

\subsection{Experimental design}

Pot experiment was conducted at the greenhouse in Zijingang campus of Zhejiang University. Rice (Oryza sativa L.) "Yongyou12" (R) was used as the test plant. Two sets of pots containing soil 1 and soil 2 without plant growth were regarded as controls (CK-S1 and CK-S2 respectively); while rice planted treatments were designed as Ri-S1 and Ri-S2. Each treatment was conducted in polluted $(P)$ and unpolluted (UP) soils, each with four replicates respectively. After surface sterilizing and washing, rice seeds were germinated in a dark growth chamber for 5 days. Three uniform and healthy seedlings were selected and then transplanted into each pots (with $15 \mathrm{~cm}$ in height and $10 \mathrm{~cm}$ in diameter) containing 1 $\mathrm{kg}$ soils with or without lindane and cultivated in the greenhouse with illumination of $12 \mathrm{~h}$, average humidity of $70 \% / 85 \%$ and temperatures of $28 / 20^{\circ} \mathrm{C}$ (day/night). All the treatments were watered every other day to maintain the logged condition.

\subsection{Sampling method}

Samples were collected at day 10,30 , and 60 after rice seedlings transfer. Detailed sampling methods for each rhizocompartment followed by Feng et al. (2019). The original root samples were vortexed in fresh PBS solution $(\mathrm{pH} 7.4)$ for $15 \mathrm{~s}$ and then centrifuged at $4000 \mathrm{~g}$ for $10 \mathrm{~min}$. The resulting slurry was defined as rhizosphere soils. The roots were then washed in PBS solution cyclically and sonicated for $30 \mathrm{~s}$ twice more. The remaining roots were washed twice with fresh PBS, ground in a bead beater, frozen in liquid nitrogen, and stored as endosphere samples. Bulk soils were sampled from $2 \mathrm{~cm}$-deep sites in unplanted pots, mix thoroughly and stored at $-80^{\circ} \mathrm{C}$.

\subsection{Chemical analyses}

Lindane was extracted by the method of accelerated solvent extraction, and determined by gas chromatography mass spectrometry (GC-MS) (Agilent 6890N, USA) according to our previous study (Feng et al., 2019). Soil pH was estimated using a pH meter (1:2.5 w/v) (Seven Excellence METTLERTOLEDO, Switzerland). The concentration of soil sulfate was detected with ion chromatography (Dionex ICS-2000, USA). $\mathrm{HCl}$-extracted $\mathrm{Fe}(\mathrm{II})$ was colorimetrically determined using a spectrophotometer (Xu et al., 2015). Concentrations of soil dissolved organic nitrogen (DON) and carbon (DOC) were detected with a TOC analyzer (Multi N/C 3100, Germany) after extracted with Milli-Q water. The capacity of root radial oxygen loss (ROL), as indicated by the content of dissolved oxygen (DO), was in situ detected by an oxygen microelectrode system (OXY25, Unisense, Aarhus, Denmark) (Li and Wang, 2013).

\subsection{DNA extraction and high-throughput sequencing}

As the manufacturer's instructions, total DNA was extracted by FastDNA SPIN kit (QBIO gene Inc., United States). The primers 515F (5'-GTGCCAGCMGCCGCGGTAA-3') and 806R (5'-GGACTACHVGGGTWTCTAAT-3') in the V4 region of bacterial 16S rRNA gene were used for PCR amplification. Paired-end sequencing (based on Illumina MiSeq platform) was conducted by Magigene Biotechnology Co., Ltd. (Guangzhou, China). The original raw sequences were deposited in the NCBI Small Read Archive (SRA) data set (the BioProject number PRJNA533710). The Quantitative Insights into Microbial Ecology (QIIME) was used for pairedend reads merging (Caporaso et al., 2010). Based on Usearch (V10.0), high-quality sequences were clustered into the same operational taxonomic units (OTUs) at the level of $97 \%$ similarities. The taxonomy of each representative OTU was classified using RDP Classifier.

Table 1 Soil basic properties

\begin{tabular}{llllllllllll}
\hline Soil type & $\mathrm{pH}$ & $\begin{array}{l}\mathrm{OM} \\
(\%)\end{array}$ & $\begin{array}{l}\mathrm{TC} \\
(\%)\end{array}$ & $\begin{array}{l}\mathrm{TN} \\
(\%)\end{array}$ & $\begin{array}{l}\mathrm{AP} \\
\left(\mathrm{mg} \mathrm{kg}^{-1}\right)\end{array}$ & $\begin{array}{l}\mathrm{AK} \\
\left(\mathrm{mg} \mathrm{kg}^{-1}\right)\end{array}$ & $\begin{array}{l}\mathrm{SO}_{4}^{2-} \\
\left(\mathrm{mg} \mathrm{kg}^{-1}\right)\end{array}$ & $\begin{array}{l}\mathrm{Fe}(\mathrm{II}) \\
\left(\mathrm{mg} \mathrm{kg}^{-1}\right)\end{array}$ & $\begin{array}{l}\text { Clay\% } \\
(\%)\end{array}$ & $\begin{array}{l}\text { Silt\% } \\
(\%)\end{array}$ & $\begin{array}{l}\text { Sand\% } \\
(\%)\end{array}$ \\
\hline Soil 1 & 6.76 & 5.44 & 1.95 & 0.21 & 3.28 & 2.15 & 15.78 & 910.3 & 36.3 & 45.3 & 18.4 \\
Soil 2 & 4.54 & 1.56 & 1.51 & 0.19 & 1.38 & 4.58 & 4.45 & 139.8 & 17.8 & 63.9 & 18.3 \\
\hline
\end{tabular}

Abbreviations: OM, organic matter; TC, total carbon; TN, total nitrogen; AP, available phosphorus; AK, available potassium. 


\subsection{Statistical analysis}

Bacterial differences among all the samples were assessed by Permutational multivariate analyses of variance (PERMANOVA) and presented by the non-metric multidimensional scaling (NMDS) analysis based on Bray-Curtis dissimilarity matrix. The adjusted $P$-values and false discovery rate (FDR) were evaluated for multiple tests of microbial communities (Benjamini and Hochberg, 1995; Peperanney et al., 2016). The microbial composition in each compartment during rice growth period was visualized with ggalluvial package. EdgeR package was used for analyzing the differential OTUs (Robinson et al., 2009). Spearman's correlations between environmental variables and microbes at genus level were shown with strong (with correlation coefficient $<-0.5$ or $>0.5$ ) and significant $(P<0.05)$ correlations. Tax4Fun package was used for metagenome functional profiling prediction using $16 \mathrm{~S}$ rRNA gene sequences (Asshauer et al., 2015). Random Forests approach for classification and regression was applied to perform taxa variation during rice developmental stages (Zhang et al., 2018). Co-occurrence ecological networks among bacteria and environmental variables were constructed based on Molecular Ecological Networks Analysis Pipeline (MENA) platform and presented using Cytoscape (v3.6) software (Deng et al., 2012). Other graphs were visualized using OriginPro 2017 and R (v 3.4.0) with pheatmap and ggplot2 packages.

\section{Results and discussion}

3.1 Root-associated microbiota varied with soil type, time of growth and rhizo-compartments

Bacterial community structure associated with the rhizocompartments of rice roots separated due to vegetative growth phase, soil types, as well as lindane pollution (Fig. 1 and Table 2). Growth time displayed a divergently spatial pattern along the first axis, with bacterial communities at day 10 samples clustering, and day 30 and 60 clustering in closer proximity (Fig. 1A). Adonis analysis indicated that the microbiota significantly differed with growth time $\left(R^{2}=0.12\right.$, $P<0.001$ ) (Table 2), in line with prior results (Chaparro et al., 2014; Walters et al., 2018). It has been suggested that growth time is the strongest factor shaping the rhizosphere commu-
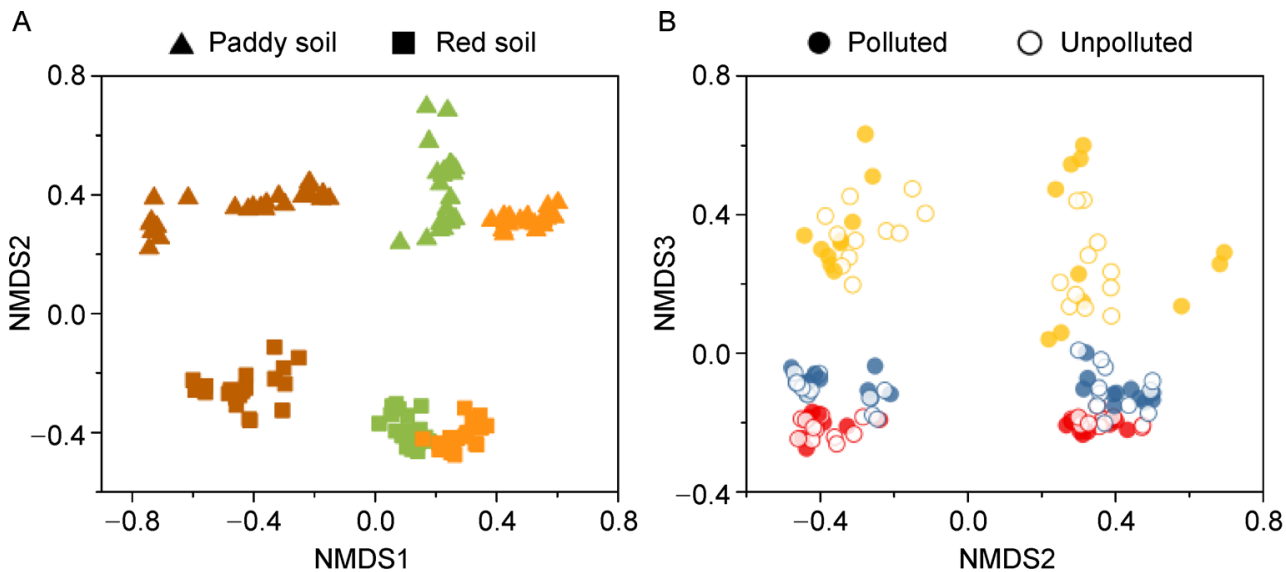

$$
\begin{array}{|l}
\text { Day } \\
\begin{array}{r}
10 \\
30 \\
60
\end{array}
\end{array}
$$

\section{Compartment}

Bulk soils

Rhizosphere

Endosphere

C

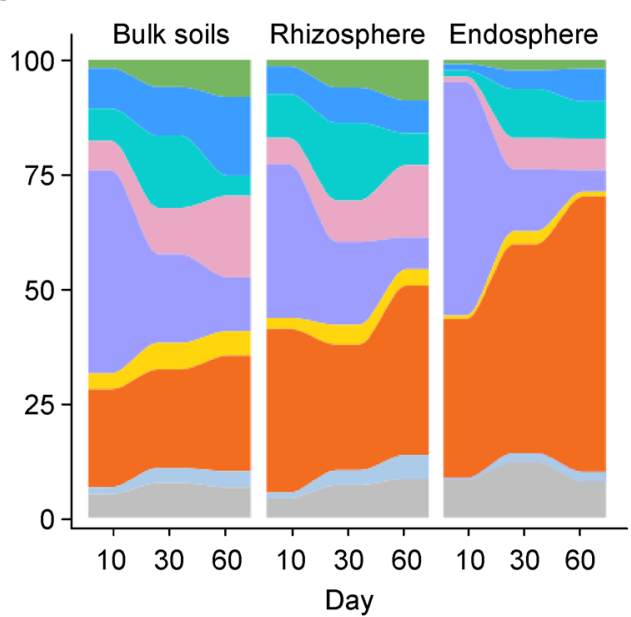

$D$

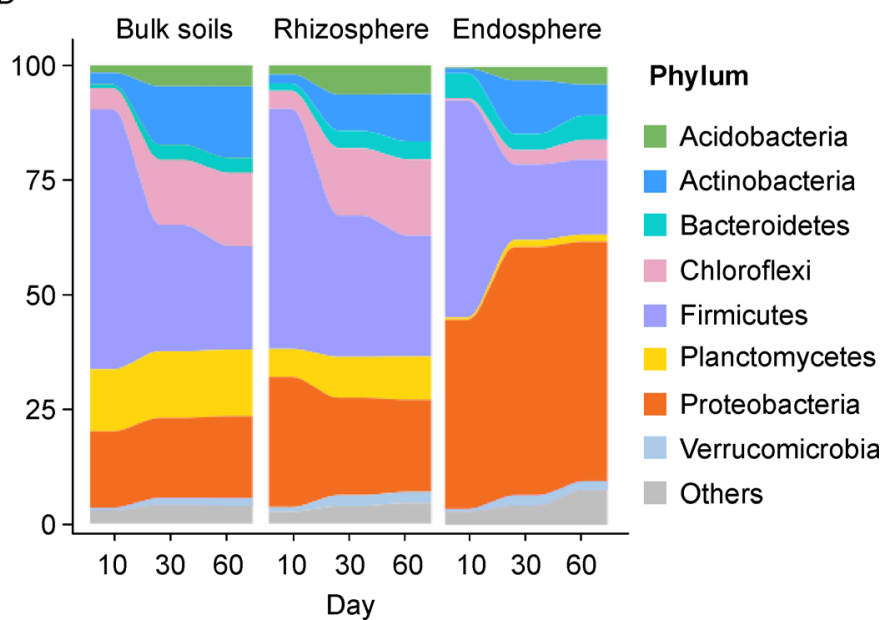

Fig. 1 The shift of microbial $\beta$-diversity $(A, B)$ and composition $(C, D)$ of bacteria in different treatments. NMDS analysis shows that the root-associated microbiota shifts with growth time in different soil types $(A)$ and influenced by rhizo-compartments and lindane pollution (B); and relative abundance of bacteria at phylum level with the growth time of rice in soil 1 (C) and soil 2 (D). 
Table 2 The impacts of variables on the microbial community described by PERMANOVA ${ }^{a}$.

\begin{tabular}{|c|c|c|c|c|c|c|c|}
\hline & $\mathrm{df}$ & SS & MS & F.Model & $R^{2}$ & $P$ value ${ }^{c}$ & \\
\hline Growth time & 1 & 4.491 & 4.4906 & 32.225 & 0.12473 & 0.001 & $* * *$ \\
\hline Pollution & 1 & 0.156 & 0.1561 & 1.120 & 0.00433 & 0.326 & \\
\hline Compartment & 2 & 4.043 & 2.0215 & 14.507 & 0.11230 & 0.001 & *** \\
\hline Soil type & 1 & 1.328 & 1.3285 & 9.533 & 0.03690 & 0.001 & $* * *$ \\
\hline Growth time $\times$ pollution $^{b}$ & 1 & 0.166 & 0.1657 & 1.189 & 0.00460 & 0.270 & \\
\hline Growth time $\times$ compartment & 2 & 1.194 & 0.5968 & 4.282 & 0.03315 & 0.001 & $* * *$ \\
\hline Pollution $\times$ compartment & 2 & 0.388 & 0.1939 & 1.391 & 0.01077 & 0.138 & \\
\hline Growth time $\times$ soil type & 1 & 3.686 & 3.6860 & 26.451 & 0.10238 & 0.001 & $* * *$ \\
\hline Pollution $\times$ soil type & 1 & 0.170 & 0.1698 & 1.219 & 0.00472 & 0.260 & \\
\hline Compartment $\times$ soil type & 2 & 1.382 & 0.6911 & 4.960 & 0.03839 & 0.001 & *** \\
\hline Growth time $\times$ pollution $\times$ compartment & 2 & 0.348 & 0.1741 & 1.250 & 0.00967 & 0.195 & \\
\hline Growth time $\times$ pollution $\times$ soil type & 1 & 0.191 & 0.1912 & 1.372 & 0.00531 & 0.167 & \\
\hline Growth time $\times$ compartment $\times$ soil type & 2 & 2.271 & 1.1353 & 8.147 & 0.06307 & 0.001 & *** \\
\hline Pollution $\times$ compartment $\times$ soil type & 2 & 0.212 & 0.1061 & 0.761 & 0.00589 & 0.767 & \\
\hline Growth time $\times$ pollution $\times$ compartment $\times$ soil type & 2 & 0.231 & 0.1156 & 0.830 & 0.00642 & 0.695 & \\
\hline Residuals & 113 & 15.747 & 0.1394 & 0.437 & & & \\
\hline Total & 136 & 36.003 & 1 & & & & \\
\hline
\end{tabular}

PERMANOVA, permutational multivariate analysis of variance; df, degree of freedom; SS, sums of squares; MS, mean square; " $\times$," interactions between environment variables; Adjusted- $P$ value: ${ }^{*}, P<0.05 ;{ }^{* *}, P<0.01 ;{ }^{* *}, P<0.001$.

nities of maize (Walters et al., 2018); and rice root microbiota undergoes the greatest changes from seedling stage to other growth stages (Chaparro et al., 2014).

Soil 1 and soil 2 separated along the second axis $\left(R^{2}=\right.$ $0.04, P<0.001$ ) (Fig. 1A and Table 2). The two soils differed in physicochemical properties (Table 1), with soil 1 having a higher $\mathrm{pH}$, organic matter content, $\mathrm{Fe}(\mathrm{II})$ and clay components compared to soil 2. Soil type was one of the most important sources of variation within the root-associated microbial communities of rice. However, it did not affect the separation between rhizosphere samples, indicating a strong recruitment influence on microbial consortia by rhizo-compartments. The root-associated microbiota of rhizo-compartments was clearly observed across the third axis, with endosphere compartment separated alone and rhizosphere community clustered close to bulk soils $\left(R^{2}=0.11, P<0.001\right)$ (Fig. $1 \mathrm{~B}$ and Table 2$)$. This was in consistent with previous results that compartment was a major factor on root-associated microbiota, with the endosphere being a more independent zone with strict microbiota selection (Edwards et al., 2015).

The interaction effects of environmental variables further emphasized that growth time, soil types and rhizo-compartments are all dominating factors affecting the microbial compositions. The combined impacts between two of these three factors or among three factors were remarkable on the microbial structure, and this was in accordance with the prior results on some typical plants $(23.7 \%, P<0.001)$ (Table 2$)$
(Bulgarelli et al., 2012; Edwards et al., 2015). Unlike the factors discussed above, lindane pollution had an insignificant influence on root-associated microbiota, neither separately nor together with other variables $(P>0.05)$ (Table 2 and Fig. 1B). Worth mentioning, to expand the pollution stress of residual lindane at the most possibility, the spiked concentration of lindane was artificially increased on purpose as compared to usually reported in the previous literatures. Even though, we still found an insignificant and much lesser impact of residual lindane on the whole microbial structure comparing with soil types, time period and compartments. This may indicate that in natural polluted soils with relatively lower concentrations, the real impact of residual OCPs for the assembly of root-associated microbiota would be finite.

The shift in relative abundance of eight main phyla was characterized in response to the driving factors (Fig. 1C and 1D). In soil 1, the relative abundance of Acidobacteria, Chloroflexi and Proteobacteria significantly increased, but Firmicutes decreased over time. The phylum of Bacteroidetes reached its peak abundance in both bulk and rhizosphere compartments at day 30 . In soil 2 , Firmicutes was the dominant phylum during all the time periods, but their relative abundance dramatically decreased from day 10 to day 60 ; while the proportions of Actinobacteria and Chloroflexi significantly increased during this growth phase. Additionally, significant difference in the composition of endosphere bacteria was shown in neither soil 1 nor soil 2 . Firmicutes 
and Proteobacteria were the most abundant phyla in rice root, with the relative abundance of Proteobacteria significantly increasing and Firmicutes decreasing over time. Combined with the previous results reported for Arabidopsis, maize, and rice, our results further proved that the microbial variation from soils into the roots were alike for most land plants (Lundberg et al., 2012; Edwards et al., 2015). Additionally, Zhang et al. (2018) revealed that rice root microbiota would be stabilized 8-10 weeks after transplanting, and subsequently undergo minor changes until ripening, indicating that different root microbes might be recruited by host plants during early growth stages.

3.2 Root microbiota as biomarkers for rice cultivation and the influence from lindane pollution

A regression of proportions of endosphere bacteria at the genus level against rice growth stage was performed to evaluate the correlations between root microbiota and rice growth. $90.1 \%$ variance of endosphere microbiota was concerned in growth time, with the minimum cross-validation error taken by 21 genera (Fig. 2 and Fig. S1). Among these sensitive biomarker taxa, genera in the phylum of Proteobacteria (e.g., Acidibacter, Halianggium, Azospirillum, Desulfovibrio, Gallionella, Klebsiella, Acinetobacter and etc.) were the most dominated, accounting for $61.9 \%$ of the biomarkers for rice cultivation, followed by 6 genera in the phylum of
Firmicutes (e.g., Gracilibater, Fonticella, Desulfitobacterium, and etc.), which were in accordance with the composition of endosphere microbiota (Fig. 2). Prior results have indicated that the variation of root microbiota was always age-sensitive and these microbes probably participated in the metabolic processes in roots with the growth of rice (Edwards et al., 2018; Zhang et al., 2018). For example, Haliangium had pathogen-antagonistic and plant-growth-promoting effects (Zhou et al., 2018); Desulfovibrio was representative sulfate reducing bacteria (Xu et al., 2018) and Gallionella was ironoxidizing like bacteria (Zecchin et al., 2017) (Fig. S1). Additionally, some sensitive depleted genera, like Klebsiella, Desulfitobacterium and Acinetobacter, had been reported to have a capacity for degrading or resisting di-(2-ethylhexyl) phthalate (DEHP), polyaromatic hydrocarbon (PAH) and pentachlorophenol (PCP) in paddy soils (Chen et al., 2018; Singha et al., 2018; Xu et al., 2018; Zhu et al., 2019) (Fig. S1). The decreased abundance of these genera suggested that a diverse set of indigenous microbes would initially colonize root endosphere, but be gradually outcompeted by other taxa, affected by environmental stresses or selected against by the host plant (Edwards et al., 2018; Zhang et al., 2018).

Rice root accumulated $0.09 \mathrm{mg}, 0.56 \mathrm{mg}$ and $1.15 \mathrm{mg}$ lindane at day 10,30 and 60 , respectively. No significant difference in accumulation was observed between two soil types $(P>0.05)$ (Table 3$)$. The high hydrophobicity of lindane resulted in their restricted transfer from rhizosphere into the

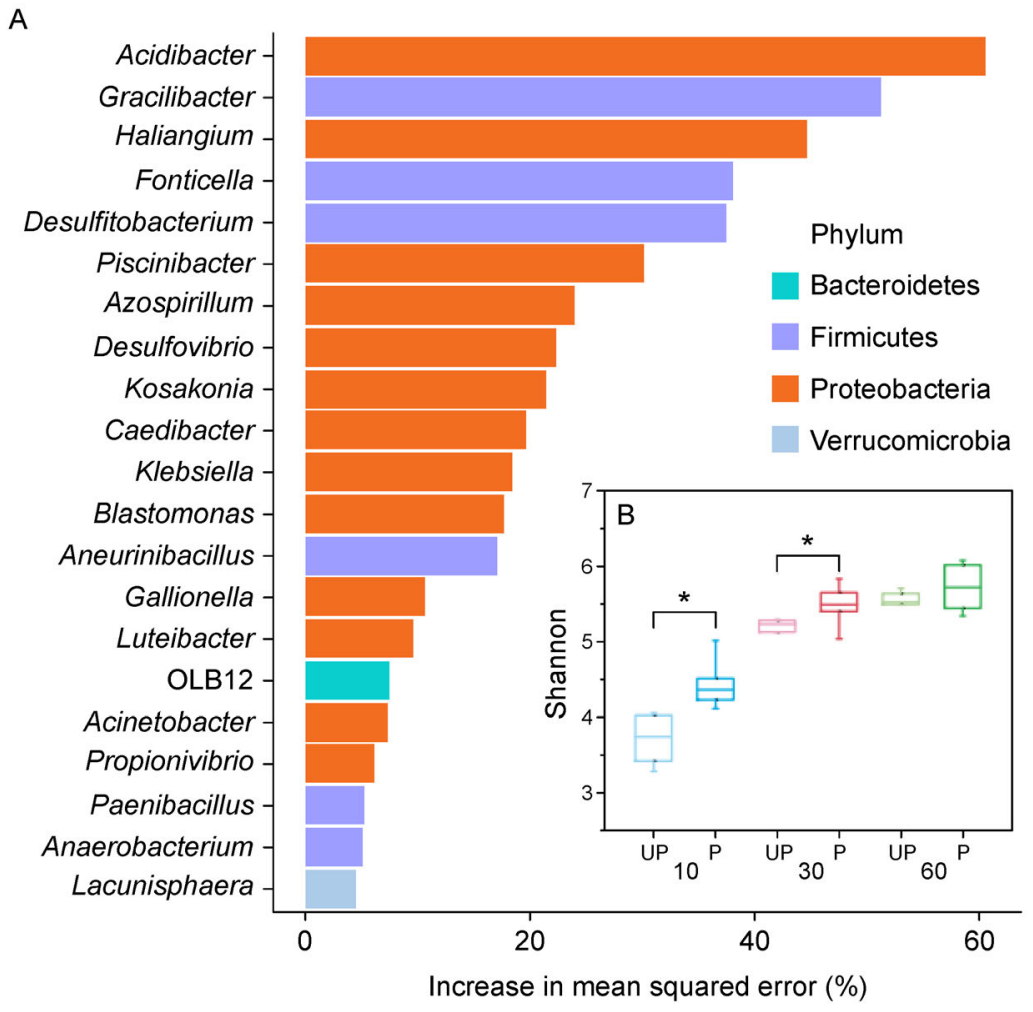

Fig. 2 Sensitive bacterial biomarkers during rice growth $(A)$ and the microbial $\alpha$-diversity between polluted and unpolluted treatments $(B)$ at the early stage of rice production. The top 21 genera were selected using Random Forests regression. UP, unpolluted treatments; $P$, polluted treatments. 
roots and shoots via apoplastic and symplastic pathways (Su and Zhu, 2007). Although lindane pollution contributed least to overall microbial variation by PERMANOVA analysis, the $\alpha-$ diversity of root endosphere was significantly higher in lindane polluted soils at day 10 and 30 (Table 2 and Fig. 2). While at day 60 , the difference was insignificant between polluted and unpolluted treatments. This suggested that lindane pollution initially increased the diversity of the endosphere microbial community before the stabilization of root microbiota; and the pollution resistant adaptability of rice was thereafter matured. Previous results have reported drought-stressed plants hosted less mature root microbiota than well-watered plants, with age-discriminant microbes being generally late colonizers (Santos-Medellín et al., 2017; Edwards et al., 2018). Luo et al. (2017) suggested that there was no consistent trend of the endosphere diversity of hyperaccumulator Sedum alfredii when planted in different heavy metals polluted soils. Therefore, we speculate that different root tissues showed plantspecific responses to contrasting environmental stresses and lindane pollution is associated with the stimulated endosphere microbiota of rice during the vegetative growth phase.

3.3 Active root-microbe-soil interactions contributed a complicated removal of lindane in rhizosphere soils of rice

During rice cultivation, the residual concentrations of lindane were higher in unplanted than in rice planted treatments at day 10 , as shown by the individual higher concentrations of both soil 1 (13.13 vs. $11.94 \mathrm{mg} \mathrm{kg}^{-1}$ ) and soil 2 (21.90 vs. 19.97 $\left.\mathrm{mg} \mathrm{kg}^{-1}\right)(P<0.05)$ (Table 3$)$. However, as rice grew gradually, the removal of lindane was significantly inhibited at day 30 and $60(P<0.05)$, with the effect more pronounced at the latter growth stage. For example, lindane removal rates were $96.6 \%$ and $96.3 \%$ at day 30 , further increased to $97.4 \%$ and $97.3 \%$ at day 60 in unplanted and rice planted treatments respectively in soil 1; while the dissipation rates were from $94.6 \%$ and $93.7 \%$ to $96.9 \%$ and $96.7 \%$ in those treatments in soil 2 (Table 3). Growing rice significantly lowered soil $\mathrm{pH}$ (6.17 vs. 5.89) $(P<0.05)$ and the concentrations of DON and $\mathrm{NH}_{4}{ }^{+}$by $14.8 \%$ and $45.1 \%$ respectively (34.11 vs. $29.06 \mathrm{mg} \mathrm{kg}^{-1}$ and 112.26 vs. $\left.61.68 \mathrm{mg} \mathrm{kg}^{-1}\right)(P<0.05)$, but increased dissolved oxygen 18-fold (5.86 vs $109.97 \mu \mathrm{mol})(P<0.05)$ and DOC by 2 fold (238.36 vs. $\left.727.84 \mathrm{mg} \mathrm{kg}^{-1}\right)(P<0.05)$, regardless of soil types. The concentrations of $\mathrm{DON}, \mathrm{NH}_{4}{ }^{+}$and $\mathrm{SO}_{4}{ }^{2-}$ decreased with rice growth, but $\mathrm{Fe}(\mathrm{II})$ increased, with no obvious differences among these variables between unpolluted and polluted treatments (Table 3 ).

The varying physicochemical properties between two soil types were thought to be a driver of the differing lindane removal due to effects on the microbial communities. The microbial diversity of soil 1 was much higher than that of soil 2 (6.38 vs. 5.38). Rice protected their roots from soil toxins by secreting radial oxygen and created a micro-aerobic zone in the rhizosphere compartment (Wang et al., 2011; Feng et al., 2019). Meanwhile, there was a much faster lindane removal rate in anoxic than in aerobic condition, thus the continuous secretion of $\mathrm{O}_{2}$ during rice growth might shift dechlorination favored anaerobic environment, then inhibiting the reductive removal of lindane in the rhizosphere; however, during the initial period of rice growth, due to a relative lower ROL capacity of young root, the suppressing effect on lindane removal would be insignificant. As such, inhibition of lindane removal by rice in both soil types only occurred at day 30 and 60 , but not day 10 . Organic compounds released by rice roots could act as carbon sources and electron donors promoting the microbial-mediated anaerobic respiration (Hayat et al., 2011). At the early growth stage of rice planting, the sufficient carbon sources in soils would satisfy the growth of both microbes and rice seedlings. This possibly counteracted the facilitating effect of root exudation, resulting in insignificant influences on dissipation of lindane between planted and unplanted treatments (Table 3). However, with the comprehensive effects of nutrient consumption and root secretion, more available carbon compounds might be preferentially allocated for microbial respiration. Therefore, the dynamic nutrient distribution at the latter growth stage of rice might potentially enhance the microbial-mediated competitive redox of some co-occurring electron acceptors like $\mathrm{SO}_{4}{ }^{2-}$ and $\mathrm{Fe}(\mathrm{III})$, and then suppress the reductive dechlorination of lindane (Xu et al., 2015; Feng et al., 2019, 2020a).

The associations between environmental factors and microbes were further evaluated by performing a significant Spearman's correlation analysis (Fig. 3). The genus Bacillus and Clostridium sp. were positively correlated with lindane dissipation and negatively related to the content of $\mathrm{Fe}(\mathrm{II})$, while Geobacter, Anaeromyxobacter and Flavisolibacter were negatively correlated; Massilia was positively correlated with the contents of $\mathrm{SO}_{4}{ }^{2-}$, DOC, DON and $\mathrm{NH}_{4}{ }^{+}$but negatively related to the content of $\mathrm{Fe}(\mathrm{II})$ (Fig. 3A). The ecological network determined the complex patterns between bacteria and environmental variables (Fig. 3B). Overall, the network was constitutive of 153 nodes and 735 edges, with the average node degree of 9.608 and path distance of 4.528 (Table S1). The phyla of Proteobacteria, Actinobacteria and Firmicutes constituted a majority of the network. The OTU258, belonging to Actinobacteria, had the maximum node degree. The $\mathrm{pH}$ was not only the most important environmental factor, but also the overriding node in the network with highest node betweenness and stress centrality. The OTUs in Actinobacteria and Firmicutes were correlated to Fe(II), and Clostridium nodes were positively correlated to lindane pollution. All of the above results strongly indicated the sensitive microbial responders in root-associated microbiota exhibited a very good relevance to soil biochemical processes occurred in the rhizosphere of rice during its growth, including lindane dissipation and other soil redox processes.

As prior results reported, in anaerobic soil environment with OCP pollution, some OCP sensitive responders, such as Bacillus, Geobacter, Clostridium and Flavisolibacter could not only utilize the available $\mathrm{Fe}(\mathrm{III})$ and $\mathrm{SO}_{4}{ }^{2-}$ as electron acceptors to participate in soil redox processes, but also use OCPs as alternative electron acceptor for their competitively 


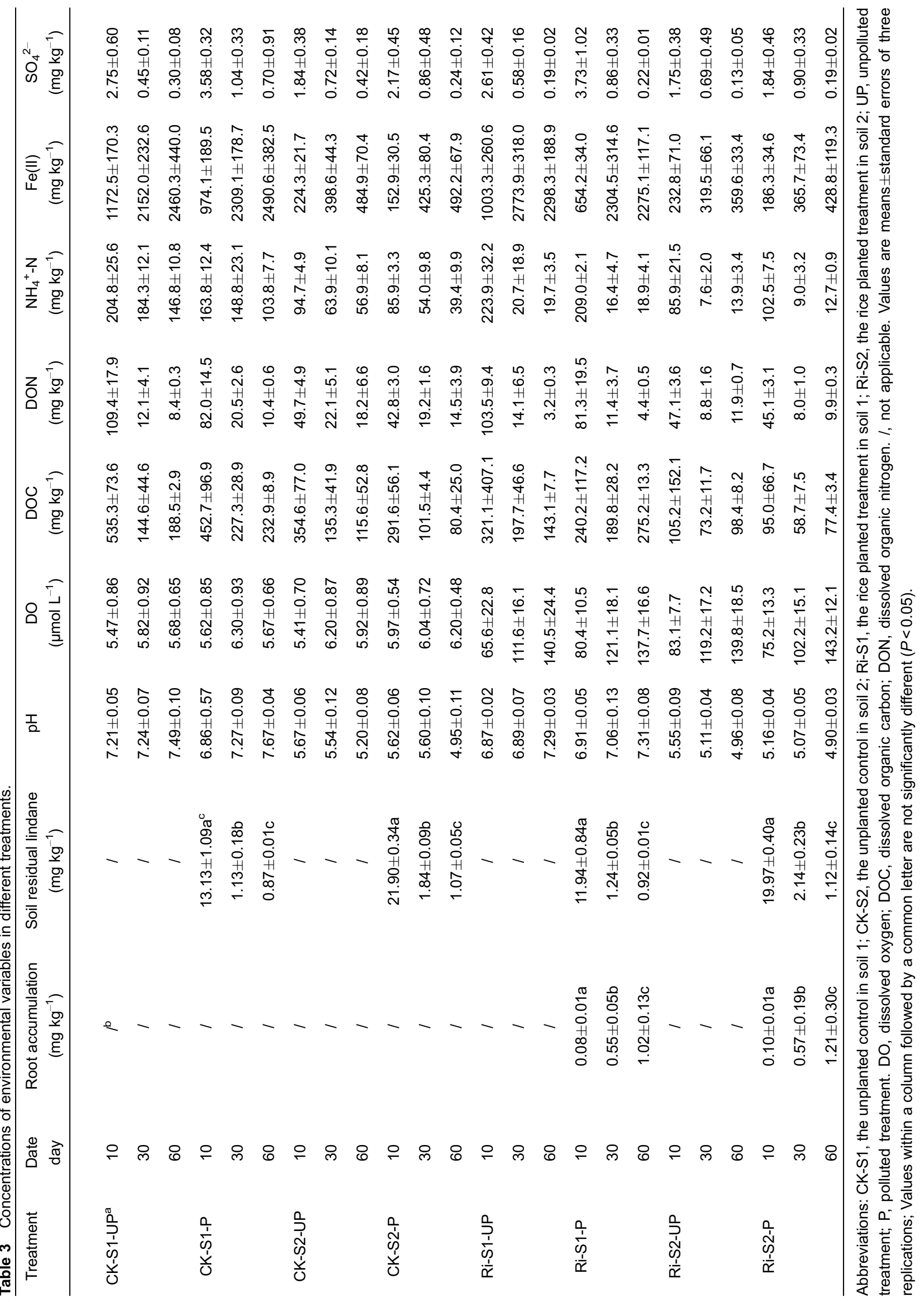


A

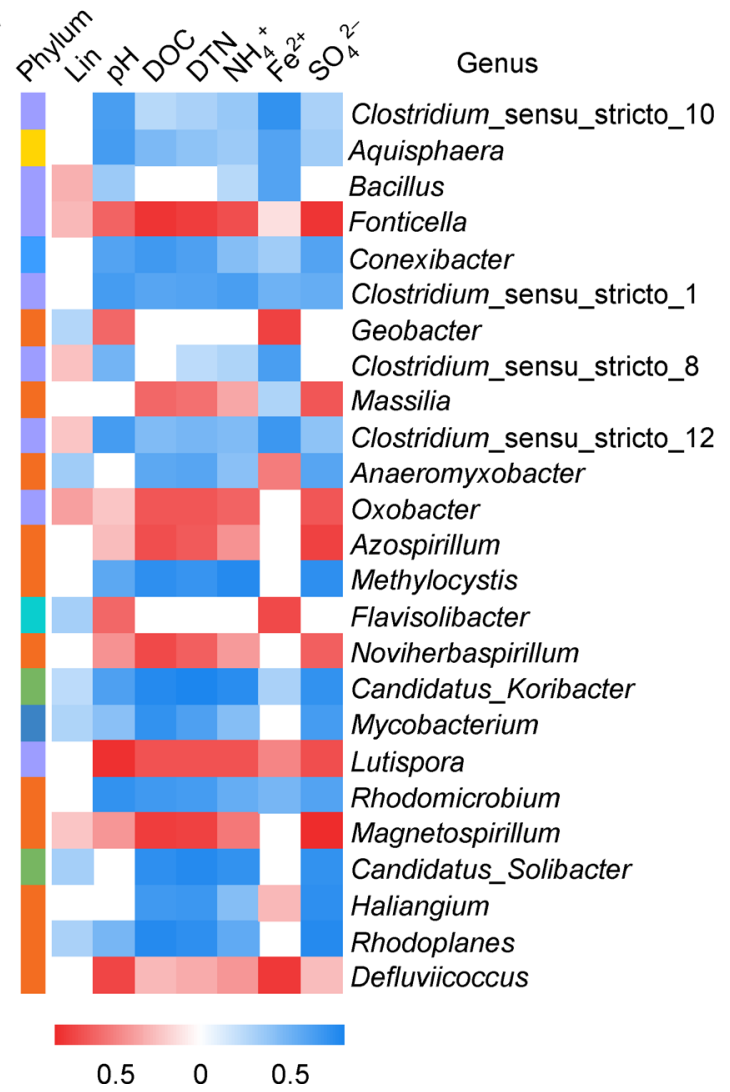

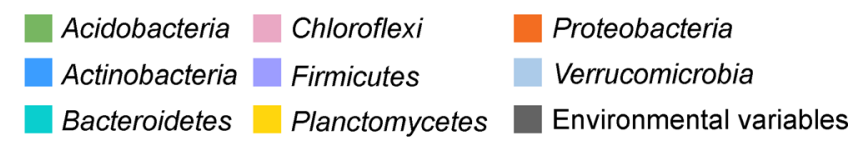

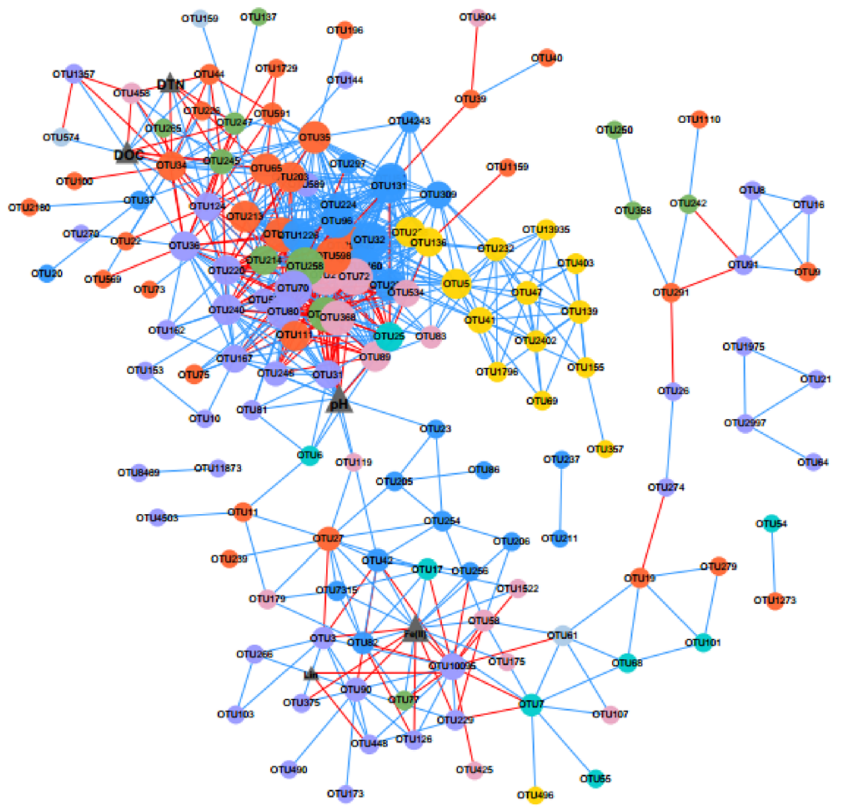

Fig. 3 Spearman's correlations $(A)$ and the network $(B)$ between bacteria taxa and environmental factors in rice rhizosphere soils. Scale bars indicate correlation coefficients. Only significant $(P<0.05)$ and strong (correlation coefficient, $r>0.5$ or $<0.5)$ correlations are shown in Fig. 3A.

dechlorinated degradation (Xu et al., 2015; Zhu et al., 2019; Feng et al., 2020a). Bachmann et al. (1988) suggested that the dissipation of $\mathrm{HCHs}$ was mediated by some sulfatereducing bacteria and inhibited under different redox conditions in soil slurries; Xu et al. $(2015,2018)$ indicated that the dechlorination of OCP was competitively coupled with $\mathrm{SO}_{4}{ }^{2-}$ reduction but synergistically with $\mathrm{Fe}(\mathrm{III})$ reduction. While the soil contained these microbes, the changes induced by rice growth inhibited lindane reductive dissipation through changes to the soil chemistry.

Our study provided insight into the complex root-microbesoil interaction in rice planted soils, and preliminarily revealed that although soil properties differentially influenced the lindane removal, microbial mediated reducing reactions, such as $\mathrm{Fe}(\mathrm{III}), \mathrm{SO}_{4}{ }^{2-}$ reduction, were fundamentally coupled with the dechlorinative removal of OCPs, such as lindane, in anaerobic conditions. These processes and corresponding functional microbes would also be affected by rice growth due to the shaping effects of root exudates and oxygen loss.

\subsection{Rhizo-compartment induced different functional responses}

Using a linear model analysis approach, we identified the specific OTUs that were remarkably inhabited in bulk soils, rhizosphere and endosphere (Fig. 4A). By contrast, the endosphere compartment shared the maximum enriched OTUs, mostly belonging to Protobacteria and Firmicutes, and accounting for over $58.9 \%$ of the total relative abundance. Lower family level analysis showed that the enrichment of these two phyla in root was driven by a predominant subset of Burkholderiaceae and Bacillaceae. Actinobacteria and Planctomycetes OTUs were mainly enriched in bulk soils and took up $5.70 \%$ of the microbial abundance. In addition, the rhizosphere compartment only had 8 OTUs with the abundance proportions of $0.78 \%$ (Fig. 4B).

Different microbiota of each rhizo-compartment would contribute to the divergent ecological functions for adaption to the change of environment. Therefore, Tax4Fun analysis was performed to compare the potentially ecological function between endosphere, rhizosphere and bulk soils (Fig. 4C). Notably, the rhizosphere was enriched for KEGG orthologs (KOs) associated with energy metabolism, signal transduction and cell growth and death, but had the lowest abundance in some metabolism processes, such as xenobiotics biodegradation, terpenoids and polyketides metabolism. These reductions were in accordance with the observed decreased lindane degradation rate in the rhizosphere soils comparing with non-planted control (Table 3 ). With a relative more abundant and diverse microbial communities, the rhizosphere 
A
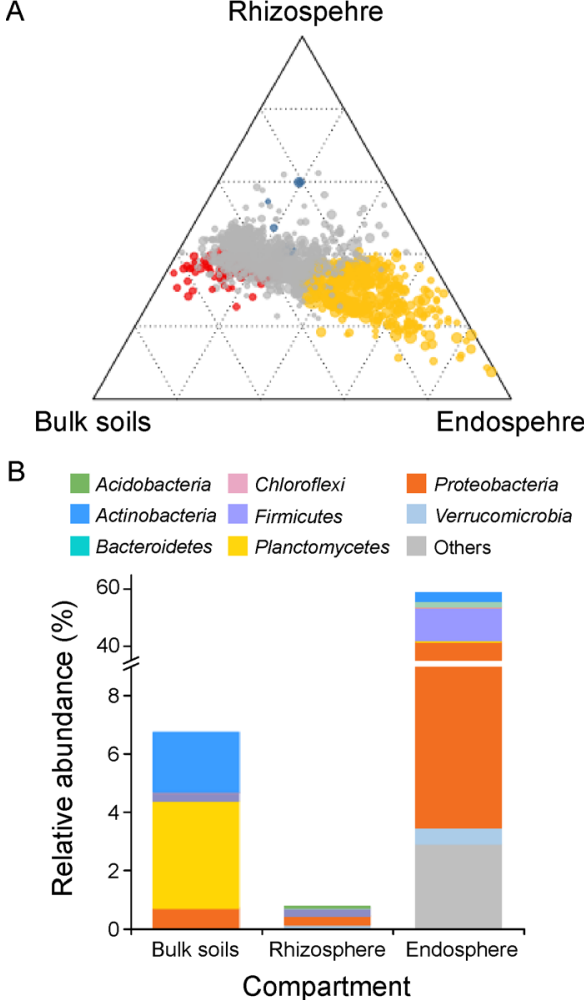

C

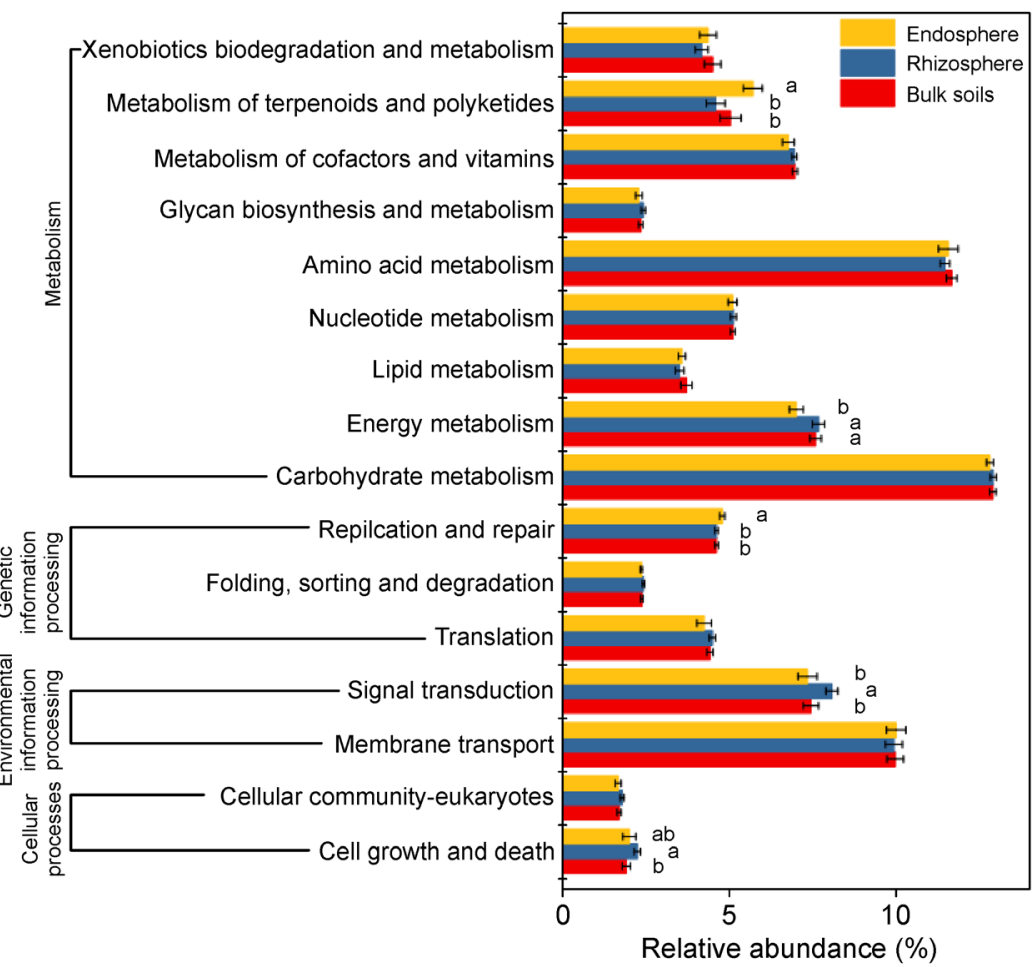

Fig. 4 Bacterial enrichment ( $A$ and $B$ ) and the predictive functional metagenome composition (mean relative abundance $>1 \%$ ) (C) in bulk soils, rhizosphere and endosphere compartment. (A): Ternary plots represent OTUs (relative abundance $>0.5 \%$ ) that significantly enriched in one compartment compared with the others (yellow in root endosphere, red in bulk soils, and blue in rhizosphere). (B): Histogram represents the total relative abundance of enriched OTUs at phylum level in each compartment. (C): Different letters show the significant differences $(P<0.05)$ by Wilcoxon test.

compartment contains more copiotrophic bacteria which have been reported important for biochemical processes and promoting electron transfer processes (Fierer et al. 2007).

The endosphere compartment had the highest abundance of KOs associated with the metabolisms of terpenoids and polyketides, replication and repair. These functions provided evidence that plant roots could enhance microbial resistance to environmental stresses (Taiz and Murphy, 1995). The phyla of Proteobacteria and Firmicutes were specifically enriched in rice root endosphere (Fig. 4A and 4B), and microbes in taxa such as Burkholderia, Geobacter, Sphingomonas, Bacillus and Clostridium (genus level) were widely reported to have the potential to contribute to lindane removal (Phillips et al., 2005; $\mathrm{Xu}$ et al., 2018). The specific enrichment of these functional microbes may contribute to the relative lower concentration of lindane in root tissues and further be conducive to the construction of the stable endosphere microbiota.

Prior studies differed from our results in reporting abundances of the KOs assigned to carbohydrate and amino acid metabolisms to be enriched in rhizosphere soils of the Sedum alfredii (Luo et al., 2017). The exudation of carboxylic acids from roots increased metal solubility and bioavailability. High abundance of membrane transporters in the endosphere pointed to a great capability for uptaking some organic matters and heavy metals (Taiz and Murphy, 1995; Luo et al., 2017). We hypothesized that although different rhizo-compartments had specific functional groupings due to the microbial community being shaped by plant effects, the variance would be differentially affected by various environmental stresses. However, due to the limits of metagenome functional prediction, more studies are necessary to focus on the accurate technical methods to excavate the ecological functions responsible for lindane removal which is coupled with soil redox processes.

\section{Conclusion}

This study revealed the assembly of root-associated microbiota of rice spatiotemporally, and clearly verified its variance significantly responded to soil type, rice growth time, and rhizo-compartment. By contrast, lindane pollution had less obvious influence on the rice growth and soil microbial communities, but mostly stimulated the construction of root endosphere microbiota at the vegetative phase which was highly dynamic during rice growth. Active rice root-soilmicrobe interactions induced a complicated soil-redoxcoupled removal of lindane, leading to an inhibition effect for 
lindane removal at the later vegetative growth phase in ricegrowth-dependent anaerobic condition. This might be mainly due to the root oxygen loss and microbial mediated cooccurring competitive electron-consuming redox processes in soils. Each rhizo-compartment owned distinct microbial communities, and therefore, presented specific ecologically functional categories. Our study provides detailed insight into the underground micro-ecological process of root-associated microbiota of rice growing in soil environment polluted by OCPs such as lindane, and their potential linkage to the natural attenuation of residual OCPs during rice vegetative growth phase. This is obviously beneficial for an improved regulation of plant growth against environmental adversity, and thereby rice production in COP polluted area.

\section{Acknowledgments}

This research was financially supported by the National Natural Science Foundation of China (41721001, 41771269), China Agriculture Research System (CARS-04), and the National Key Research and Development Program of China (2016YFD0800207).

\section{References}

Asshauer, K.P., Wemheuer, B., Daniel, R., Meinicke, P., 2015 Tax4Fun: predicting functional profiles from metagenomic 16S rRNA data. Bioinformatics (Oxford, England) 31, 2882-2884.

Benjamini, Y., Hochberg, Y., 1995. Controlling the false discovery ratea practical and powerful approach to multiple testing. Journal of the Royal Statistical Society. Series A, (Statistics in Society) 57, 289 300.

Bulgarelli, D., Rott, M., Schlaeppi, K., Ver Loren Van Themaat, E., Ahmadinejad, N., Assenza, F., Rauf, P., Huettel, B., Reinhardt, R., Schmelzer, E., Peplies, J., Gloeckner, F.O., Amann, R., Eickhorst, T., Schulze-Lefert, P., 2012. Revealing structure and assembly cues for Arabidopsis root-inhabiting bacterial microbiota. Nature 488, 91-95.

Caporaso, J.G., Kuczynski, J., Stombaugh, J., Bittinger, K., Bushman, F.D., Costello, E.K., Fierer, N., Pena, A.G., Goodrich, J.K., Gordon, J.I., Huttley, G.A., Kelley, S.T., Knights, D., Koenig, J.E., Ley, R.E., Lozupone, C.A., McDonald, D., Muegge, B.D., Pirrung, M., Reeder, J., Sevinsky, J.R., Tumbaugh, P.J., Walters, W.A., Widmann, J., Yatsunenko, T., Zaneveld, J., Knight, R., 2010. QIIME allows analysis of high-throughput community sequencing data. Nature Methods 7, 335-336.

Castrillo, G., Teixeira, P.J.P.L., Paredes, S.H., Law, T.F., de Lorenzo, L., Feltcher, M.E., Finkel, O.M., Breakfield, N.W., Mieczkowski, P., Jones, C.D., Paz-Ares, J., Dangl, J.L., 2017. Root microbiota drive direct integration of phosphate stress and immunity. Nature 543, 513-518.

Chaparro, J.M., Badri, D.V., Vivanco, J.M., 2014. Rhizosphere microbiome assemblage is affected by plant development. ISME Journal 8, 790-803.

Chen, L., Ran, Y., Xing, B., Mai, B., He, J., We, X., Fu, J., Sheng, G.,
2015. Content and source of polycyclic aromatic hydrocarbons and organochlorine pesticides in vegetable soils of Guangzhou, China. Chemosphere 60, 879-890.

Chen, X., Wu, Y., Huang, X., Lü, H., Zhao, H., Mo, C., Li, H., Cai, Q., Wong, M., 2018. Variations in microbial community and di-(2ethylhexyl) phthalate (DEHP) dissipation in different rhizospheric compartments between low- and high-DEHP accumulating cultivars of rice (Oryza sativa L.). Ecotoxicology and Environmental Safety $163,567-576$.

Chouychai, W., Kruatrachue, M., Lee, H., 2015. Effect of plant growth regulators on phytoremediation of hexachlorocyclohexane-contaminated soil. International Journal of Phytoremediation 17, 1053-1059.

Dai, Z.M., Su, W.Q., Chen, H.H., Albert, B., Zhao, H.C., Yu, M.J., Yu, L., Philip, C.B., Christopher, W.S., Scott, X.C., Xu, J.M., 2018. Long-term nitrogen fertilization decreases bacterial diversity and favors the growth of Actinobacteria and Proteobacteria in agroecosystems across the globe. Global Change Biology 24, 34523461.

Deng, Y., Jiang, Y.H., Yang, Y., He, Z., Luo, F., Zhou, J., 2012. Molecular ecological network analyses. BMC Bioinformatics 13, 113.

Edwards, J., Johnson, C., Santos-Medellín, C., Lurie, E., Podishetty, N.K., Bhatnagar, S., Eisen, J.A., Sundaresan, V., 2015. Structure, variation, and assembly of the root-associated microbiomes of rice. Proceedings of the National Academy of Sciences of the United States of America 112, E911-E920.

Edwards, J.A., Santos-Medellín, C.M., Liechty, Z.S., Nguyen, B., Lurie, E., Eason, S., Phillips, G., Sundaresan, V., 2018. Compositional shifts in root-associated bacterial and archaeal microbiota track the plant life cycle in field-grown rice. PLoS Biology 16 , e2003862.

Feng, J.Y., Shentu, J., Zhu, Y.J., Tang, C.X., He, Y., Xu, J.M., 2020a. Crop-dependent root-microbe-soil interactions induce contrasting natural attenuation of organochlorine lindane in soils. Environmental Pollution 257, 113580.

Feng, J.Y., Xu, Y., Ma, B., Tang, C.X., Brookes, P.C., He, Y., Xu, J.M., 2019. Assembly of root-associated microbiomes of typical rice cultivars in response to lindane pollution. Environment International 131, 104975.

Feng, J.Y., Zhu, Y.J., Shentu, J., Lu, Z.J., He, Y., Xu, J.M., 2020. 2020 b. Pollution adaptive responses of root-associated microbiomes induced the promoted but different attenuation of soil residual lindane. Science of the Total Environment 732, 139170.

Fierer, N., Bradford, M.A., Jackson, R.B., 2007. Toward an ecological classification of soil bacteria. Ecology 6, 1354-1364.

Hayat, T., Ding, N., Ma, B., He, Y., Shi, J., Xu, J., 2011. Dissipation of pentachlorophenol in the aerobic-anaerobic interfaces established by the rhizosphere of rice (L.) root. Journal of Environmental Quality 40, 1722-1729.

Hua, X., Shan, Z., 1996. The production and application of pesticides and factor analysis of their pollution in China. Advances in Environmental Sciences 4, 33-45.

Kögel-Knabner, I., Amelung, W., Cao, Z., Fiedler, S., Frenzel, P., Jahn, R., Kalbitz, K., Kölbl, A., Schloter, M., 2010. Biogeochemistry of paddy soils. Geoderma 157, 1-14. 
Li, Y., Wang, X., 2013. Root-induced changes in radial oxygen loss, rhizosphere oxygen profile, and nitrification of two rice cultivars in Chinese red soil regions. Plant and Soil 365, 115-126.

Lundberg, D.S., Lebeis, S.L., Paredes, S.H., Yourstone, S., Gehring, J., Malfatti, S., Tremblay, J., Engelbrektson, A., Kunin, V., Rio, T.G. D., Edgar, R.C., Eickhorst, T., Ley, R.E., Hugenholtz, P., Tringe, S. G., Dangl, J.L., 2012. Defining the core Arabidopsis thaliana root microbiome. Nature 488, 86-90.

Luo, J., Tao, Q., Wu, K., Li, J., Qian, J., Liang, Y., Yang, X., Li, T., 2017. Structural and functional variability in root-associated bacterial microbiomes of $\mathrm{Cd} / \mathrm{Zn}$ hyperaccumulator Sedum alfredii. Applied Microbiology and Biotechnology 101, 7961-7976.

Niu, L., Xu, C., Yao, Y., Liu, K., Yang, F., Tang, M., Liu, W., 2013. Status, influences and risk assessment of hexachlorocyclohexanes in agricultural soils across China. Environmental Science \& Technology 47, 12140-12147.

Peperanney, C., Campbell, A.N., Koechli, C.N., Berthrong, S., Buckley, D.H., 2016. Unearthing the ecology of soil microorganisms using a high resolution DNA-SIP approach to explore cellulose and xylose metabolism in soil. Frontiers in Microbiology 7, 703.

Phillips, T.M., Seech, A.G., Lee, H., Trevors, J.T., 2005. Biodegradation of hexachlorocyclohexane $(\mathrm{HCH})$ by microorganisms. Biodegradation 16, 363-392.

Poolpak, T., Pokethitiyook, P., Kruatrachue, M., Arjarasirikoon, U., Thanwaniwat, N., 2008. Residue analysis of organochlorine pesticides in the Mae Klong river of Central Thailand. Journal of Hazardous Materials 156, 230-239.

Rani, R., Usmani, Z., Gupta, P., Avantika, C., Aakankshya, D., Vipin, K., 2018. Effects of organochlorine pesticides on plant growthpromoting traits of phosphate-solubilizing rhizobacterium, Paenibacillus sp. IITISM08. Environmental Science and Pollution Research International 25, 5668-5680.

Robinson, M.D., McCarthy, D.J., Smyth, G.K., 2009. edgeR: a Bioconductor package for differential expression analysis of digital gene expression data. Bioinformatics (Oxford, England) 26, 139 140.

Salam, J.A., Hatha, M.A.A., Das, N., 2017. Microbial-enhanced lindane removal by sugarcane (Saccharum officinarum) in doped soil-applications in phytoremediation and bioaugmentation. Journal of Environmental Management 193, 394-399.

Santos-Medellín, C., Edwards, J., Liechty, Z., Nguyen, B., Sundaresan, V., 2017. Drought stress results in a compartment-specific restructuring of the rice root-associated microbiomes. mBio 8 , e00764 e17.

Singha, L.P., Sinha, N., Pandey, P., 2018. Rhizoremediation prospects of polyaromatic hydrocarbon degrading rhizobacteria, that facilitate glutathione and glutathione-S-transferase mediated stress response, and enhance growth of rice plants in pyrene contaminated soil. Ecotoxicology and Environmental Safety 164, 579 588.

Su, Y., Zhu, Y., 2007. Transport mechanisms for the uptake of organic compounds by rice (Oryza sativa) roots. Environmental Pollution 148, 94-100.

Taiz, L., Murphy, A., 1995. Comparison of metallothionein cene expression and nonprotein thiols in ten Arabidopsis ecotypes.
Plant Physiology 109, 945-954.

Tang, X., Zhang, R., Zhang, Q., Wang, W., 2016. Dehydrochlorination mechanism of $\gamma$-hexachlorocyclohexane degraded by dehydrochlorinase LinA from Sphingomonas paucimobilis UT26. RSC Advances 6, 4183-4192.

Walters, W.A., Jin, Z., Youngblut, N., Wallace, J.G., Sutter, J., Zhang, W., González-Peña, A., Peiffer, J., Koren, O., Shi, Q., Knight, R., Glavina Del Rio, T., Tringe, S.G., Buckler, E.S., Dangl, J.L., Ley, R. E., 2018. Large-scale replicated field study of maize rhizosphere identifies heritable microbes. Proceedings of the National Academy of Sciences of the United States of America 115, 73687373.

Wang, M., Chen, A., Wong, M., Quu, R., Cheng, H., Ye, Z., 2011. Cadmium accumulation in and tolerance of rice (Oryza sativa L.) varieties with different rates of radial oxygen loss. Environmental Pollution 159, 1730-1736.

Wu, C., Ye, Z., Li, H., Wu, S., Deng, D., Zhu, Y., Wong, M., 2012. Do radial oxygen loss and external aeration affect iron plaque formation and arsenic accumulation and speciation in rice? Journal of Experimental Botany 63, 2961-2970.

Xu, Y., Ge, Y., Song, J.X., Rensing, C., 2020b. Assembly of rootassociated microbial community of typical rice cultivars in different soil types. Journal of Hazardous Materials 56, 249-260.

Xu, Y., He, Y., Zhang, Q., Xu, J., Crowley, D., 2015. Coupling between pentachlorophenol dechlorination and soil redox as revealed by stable carbon isotope, microbial community structure, and biogeochemical data. Environmental Science \& Technology 49, 5425-5433.

Xu, Y., Liu, J.Q., Cai, W.S., Feng, J.Y., Lu, Z., Wang, H.Z., Franks, A. E., Tang, C.X., He, Y., Xu, J.M., 2020a. Dynamic processes in conjunction with microbial response to disclose the biochar effect on pentachlorophenol degradation under both aerobic and anaerobic conditions. Journal of Hazardous Materials 384, 121503.

Xu, Y., Xue, L., Ye, Q., Franks, A.E., Zhu, M., Feng, X., Xu, J., He, Y., 2018. Inhibitory effects of sulfate and nitrate reduction on reductive dechlorination of PCP in a flooded paddy soil. Frontiers in Microbiology 9, 9.

Xue, L., Feng, X., Xu, Y., Li, X., Zhu, M., Xu, J., He, Y., 2017. The dechlorination of pentachlorophenol under a sulfate and iron reduction co-occurring anaerobic environment. Chemosphere 182, 166-173.

Zecchin, S., Corsini, A., Martin, M., Romani, M., Beone, G.M., Zanchi, R., Zanzo, E., Tenni, D., Fontanella, M.C., Cavalca, L., 2017. Rhizospheric iron and arsenic bacteria affected by water regime: Implications for metalloid uptake by rice. Soil Biology \& Biochemistry 106, 129-137.

Zhang, A., Liu, W., Yuan, H., Zhou, S., Su, Y., Li, Y., 2011. Spatial distribution of hexachlorocyclohexanes in agricultural soils in Zhejiang Province, China, and correlations with elevation and temperature. Environmental Science \& Technology 45, 63036308.

Zhang, J., Liu, Y., Zhang, N., Hu, B., Jin, T., Xu, H., Qin, Y., Yan, P., Zhang, X., Guo, X., Hui, J., Cao, S., Wang, X., Wang, C., Wang, H., Qu, B., Fan, G., Yuan, L., Garrido-Oter, R., Chu, C., Bai, Y., 2019. NRT1.1B is associated with root microbiota composition and 
nitrogen use in field-grown rice. Nature Biotechnology 37, 676684.

Zhang, J., Zhang, N., Liu, Y.X., Zhang, X., Hu, B., Qin, Y., Xu, H., Wang, H., Guo, X., Qian, J., Wang, W., Zhang, P., Jin, T., Chu, C., Bai, Y., 2018. Root microbiota shift in rice correlates with resident time in the field and developmental stage. Science China. Life Sciences 61, 613-621.

Zhou, X., Zhang, J., Pan, D., Ge, X., Jin, X., Chen, S., Wu, F., 2018. p-
Coumaric can alter the composition of cucumber rhizosphere microbial communities and induce negative plant-microbial interactions. Biology and Fertility of Soils 54, 363-372.

Zhu, M., Zhang, L., Franks, A.E., Feng, X., Brookes, P.C., Xu, J., He, Y., 2019. Improved synergistic dechlorination of PCP in flooded soil microcosms with supplementary electron donors, as revealed by strengthened connections of functional microbial interactome. Soil Biology \& Biochemistry 136, 107515. 